\title{
Mechanism Underlying the Onset of Internal Blue Discoloration in Japanese Radish (Raphanus sativus) Roots
}

\author{
Katsunori Teranishi* ${ }^{* \dagger}$, Nagata Masayasu*, and Daisuke Masuda ${ }^{\S}$
}

${ }^{\dagger}$ Graduate School of Bioresources, Mie University, 1577 Kurimamachiya, Tsu, Mie, Japan

${ }^{\ddagger}$ Food Research Institute, National Agriculture and Food Research Organization, 2-1-12 Kannondai,

Tsukuba, Ibaraki, Japan

${ }^{\S}$ Sand Dune Agricultural Research Center, Agricultural Experiment Station, Ishikawa

Agriculture and Forestry Research Center, I 5-2 Uchihisumi, Kahoku, Ishikawa, Japan

Corresponding author Address:

Graduate School of Bioresources, Mie University, 1577 Kurimamachiya, Tsu, Mie, Japan.

Tel: +81-59-231-9615; fax: +81-59-231-9615. E-mail address: teranisi@bio.mie-u.ac.jp. 


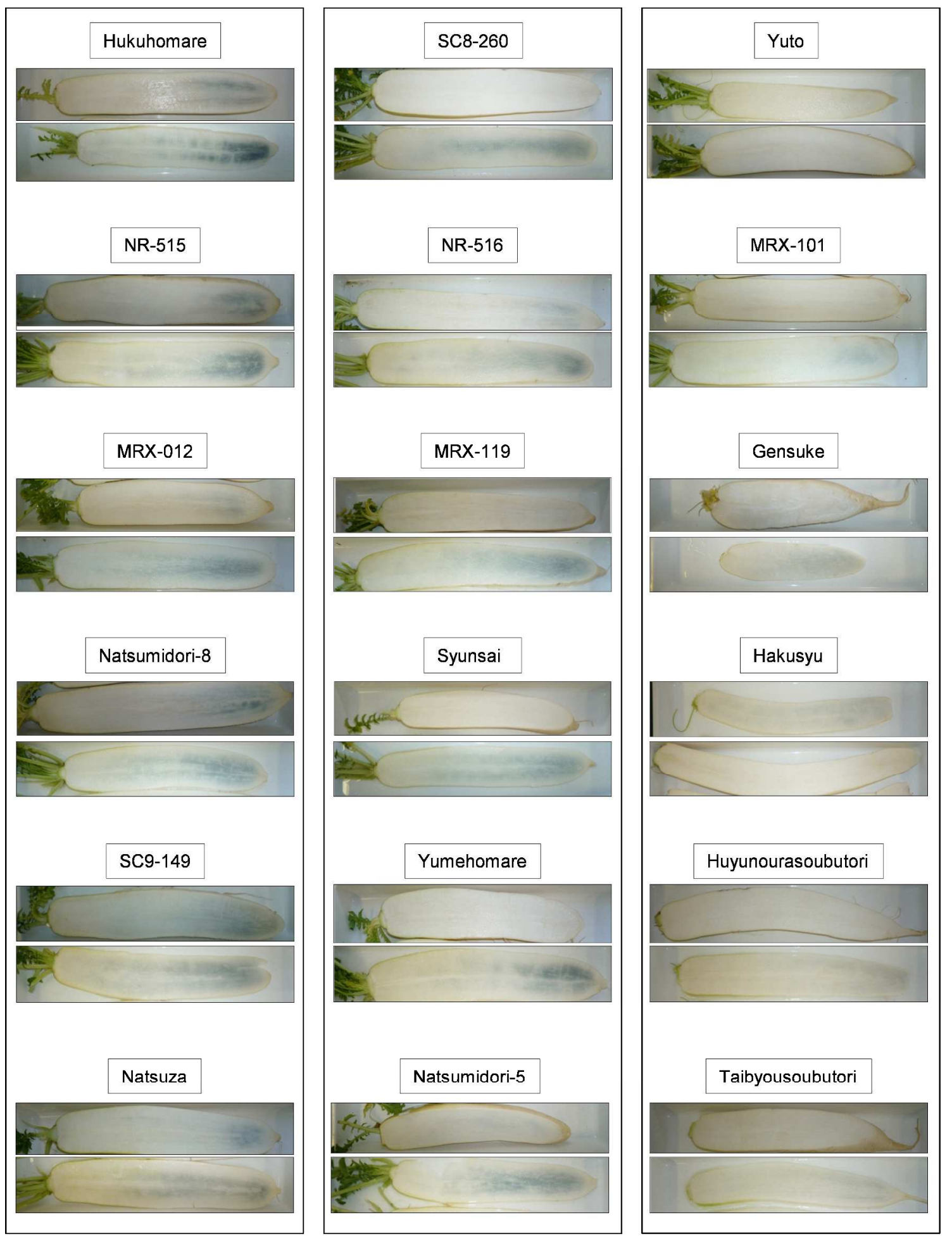

Figure S1. Three groups of blue discoloration from 18 cultivars of Japanese radish roots. Upper photos for each cultivar: after storage at $20{ }^{\circ} \mathrm{C}$ for 4 day. Bottom photos for each 
cultivar: after soaking in $0.29 \mathrm{M}$ aqueous $\mathrm{H}_{2} \mathrm{O}_{2}$ solution at room temperature for $10 \mathrm{~min}$. Left group is for cultivars that showed the discoloration with both treatments. Center group is for cultivars that showed no discoloration with storage at $20{ }^{\circ} \mathrm{C}$ and the discoloration with the $\mathrm{H}_{2} \mathrm{O}_{2}$ treatment. Right group is for cultivars that showed little or no blue discoloration with both treatments. 